\title{
Regulatory Patterns of Crp on Monensin Biosynthesis in Streptomyces cinnamonensis
}

\author{
Chun-Yan Lin ${ }^{1,+}$, Yue Zhang ${ }^{1,2,+}$, Ji-Hua Wu ${ }^{1}$, Rong-Hui Xie ${ }^{1}$, Jianjun Qiao ${ }^{1,3}$ \\ and Guang-Rong Zhao ${ }^{1,3, *}$ \\ 1 Frontier Science Center for Synthetic Biology and Key Laboratory of Systems Bioengineering \\ (Ministry of Education), School of Chemical Engineering and Technology, Tianjin University, \\ Tianjin 300350, China; lcy87828@126.com (C.-Y.L.); zhangy@tib.cas.cn (Y.Z.); 18846321904@163.com (J.-H.W.); \\ rhxie0811@126.com (R.-H.X.); jianjunq@tju.edu.cn (J.Q.) \\ 2 Present address: Tianjin Institute of Industrial Biotechnology, Chinese Academy of Sciences, \\ Tianjin 300308, China \\ 3 SynBio Research Platform, Collaborative, Innovation Center of Chemical Science and Engineering (Tianjin), \\ Tianjin 300072, China \\ * Correspondence: grzhao@tju.edu.cn; Tel.: +86-22-8535-6580; Fax: +86-22-2740-3389 \\ + These authors contributed equally to this work.
}

Received: 14 January 2020; Accepted: 15 February 2020; Published: 17 February 2020

check for updates

\begin{abstract}
Monensin, produced by Streptomyces cinnamonensis, is a polyether ionophore antibiotic widely used as a coccidiostat and a growth-promoting agent in agricultural industry. In this study, cyclic AMP receptor protein (Crp), the global transcription factor for regulation of monensin biosynthesis, was deciphered. The overexpression and antisense RNA silencing of crp revealed that Crp plays a positive role in monensin biosynthesis. RNA sequencing analysis indicated that Crp exhibited extensive regulatory effects on genes involved in both primary metabolic pathways and the monensin biosynthetic gene cluster (mon). The primary metabolic genes, including acs, pckA, $a c c B, a c d H, a t o B, m u t B$, epi and $c c r$, which are pivotal in the biosynthesis of monensin precursors malonyl-CoA, methylmalonyl-CoA and ethylmalonyl-CoA, are transcriptionally upregulated by Crp. Furthermore, Crp upregulates the expression of most mon genes, including all PKS genes (monAI to monAVIII), tailoring genes (monBI-monBII-monCI, monD and monAX) and a pathway-specific regulatory gene $(m o n R I)$. Enhanced precursor supply and the upregulated expression of mon cluser by Crp would allow the higher production of monensin in S. cinnamonensis. This study gives a more comprehensive understanding of the global regulator Crp and extends the knowledge of Crp regulatory mechanism in Streptomyces.
\end{abstract}

Keywords: Streptomyces cinnamonensis; monensin biosynthesis; Crp; transcription factor; transcription regulation; RNA sequencing

\section{Introduction}

Streptomyces bacteria are a particularly abundant source of natural products, providing more than half of medically valuable antimicrobial and anticancer drugs [1]. The antibiotic biosynthesis in Streptomyces is subject to strict control at the transcriptional level by pathway-specific regulators and global regulators [2]. The pathway-specific factors regulate the expression of antibiotic biosynthetic gene cluster and the regulatory patterns on antibiotic biosynthesis are comprehensively established [3]. However, the global regulators exhibit broader effects on both primary and secondary metabolism [4], which might directly or indirectly contribute to the antibiotic biosynthesis in streptomycetes.

The cyclic AMP receptor protein (Crp) is a well-known global factor for regulation of sugar metabolism in Escherichia coli [5-7]. The nutrient-related effects of Crp on morphology and cell 
metabolism have been reported in actinomycetes [8]. The crp deletion led to the acceleration of sporulation with reduced and delayed germination and other morphological developmental defects in S. coelicolor [9-11]. In addition to the regulation involved in primary metabolism, previous studies showed that the overexpression of the crp gene improved the biosynthesis of actinorhodin, undecylprodigiosin and calcium-dependent antibiotic in S. coelicolor and erythromycin in Saccharopolyspora erythraea [12,13]. Thus, CRP also plays important regulatory roles in antibiotic biosynthesis.

Monensin, a polyether ionophore antibiotic produced by Streptomyces cinnamonensis, is used as a growth-promoting agent for cattle and a coccidiostat for chickens in agricultural industry [14]. In recent years, monensin has been identified as a potential anticancer candidate drug because of its in vitro inhibitory effects on proliferation of several types of cancer cells [15-17]. Monensin biosynthetic gene cluster (mon) has been sequenced, and the functions have been partially characterized $[18,19]$. Eight separate type I polyketide synthases (PKSs) (MonAI to MonAVIII) were proposed to be responsible for assembling short chain acyl-CoA precursors into carbon backbone of monensin molecule [20]. The tailoring enzymes MonBI, MonBII and MonCI are involved in the oxidative cyclization of a linear polyketide intermediate [21-23]; MonD hydroxylases at C-26, and MonE methylates hydroxyl group at C-3 [24]. Thioesterase releases the final monensin from polyketide synthase [25].

Our laboratory focuses on the transcriptional factors for regulation of monensin biosynthesis. Our previous study showed that MonRI, MonRII and MonH are three positive pathway-specific regulators and that they cooperatively control mon genes in monensin biosynthesis [26]. The global factor DasR positively controls monensin biosynthesis via regulating both pathway-specific regulatory gene monRII and function genes such as monAIX, monE and monT [27]. In this study, we endeavored to decipher Crp regulatory patterns in monensin biosynthesis and identified several key genes in acyl-CoA supply pathways and most of the mon genes that are subjected to Crp regulation.

\section{Materials and Methods}

\subsection{Bacterial Strains, Chemicals and Growth Conditions}

The parental strain S. cinnamonensis ST021, which is derived from S. cinnamonensis ATCC15413, was deposited in China General Microbiological Culture Collection Center (CGMCC, Beijing, China) under the accession number CGMCC 7.240. Modified Gauze's Medium No.1 (20 g/L starch, $5 \mathrm{~g} / \mathrm{L}$ soybean flour, $1 \mathrm{~g} / \mathrm{L} \mathrm{KNO}_{3}, 0.5 \mathrm{~g} / \mathrm{L} \mathrm{NaCl}, 0.5 \mathrm{~g} / \mathrm{L} \mathrm{MgSO}_{4}, 0.5 \mathrm{~g} / \mathrm{L} \mathrm{K}_{2} \mathrm{HPO}_{4}, 0.01 \mathrm{~g} / \mathrm{L} \mathrm{FeSO}_{4}, 18 \mathrm{~g} / \mathrm{L}$ agar, $\mathrm{pH}$ adjusted to 7.4) was employed for the growth and sporulation of $S$. cinnamonensis strains for 7 days under $30^{\circ} \mathrm{C}$. For fermentation, $25 \mathrm{~mL}$ of seed medium $(16 \mathrm{~g} / \mathrm{L}$ soybean flour, $2.5 \mathrm{~g} / \mathrm{L}$ yeast extract, $20 \mathrm{~g} / \mathrm{L}$ dextrin, $5 \mathrm{~g} / \mathrm{L}$ glucose, $1.2 \mathrm{~g} / \mathrm{L} \mathrm{CaCO}_{3}, \mathrm{pH}$ adjusted to 6.8) was contained in a 250-mL flask and cultivated for $24 \mathrm{~h}$ under $30^{\circ} \mathrm{C}$ and $250 \mathrm{rpm}$. Fermentation medium $(25 \mathrm{~g} / \mathrm{L}$ soybean oil, $36.7 \mathrm{~g} / \mathrm{L}$ glucose, $16 \mathrm{~g} / \mathrm{L}$ soybean flour, $3 \mathrm{~g} / \mathrm{L} \mathrm{CaCO} 3,2 \mathrm{~g} / \mathrm{L} \mathrm{Na}_{2} \mathrm{SO}_{4}, 2.2 \mathrm{~g} / \mathrm{L} \mathrm{NaNO}{ }_{3}, 0.1 \mathrm{~g} / \mathrm{L} \mathrm{FeSO}_{4}, 0.07 \mathrm{~g} / \mathrm{L} \mathrm{Al}_{2}$ $\left(\mathrm{SO}_{4}\right)_{3}, 0.075 \mathrm{~g} / \mathrm{L} \mathrm{K}{ }_{2} \mathrm{HPO}_{4}, 0.01 \mathrm{~g} / \mathrm{L}$ vitamin $\mathrm{C}, \mathrm{pH}$ adjusted to 6.8$)$ was introduced with $8 \%$ volume of seed culture and cultivated at $30{ }^{\circ} \mathrm{C}$ and $250 \mathrm{rpm}$ for 10-12 days. All media were sterilized by autoclaving at $121^{\circ} \mathrm{C}$ for $20 \mathrm{~min}$ before use. When necessary, antibiotics were added in the following concentrations: $25 \mu \mathrm{g} / \mathrm{mL}$ hygromycin, $20 \mu \mathrm{g} / \mathrm{mL}$ nalidixic acid.

\subsection{Plasmid Constructions for Gene Overexpression and Silencing}

The plasmids and strains used in this study are listed in Table 1. The primers are listed in Supplementary Table S1. Gene manipulation of Escherichia coli and Streptomyces were performed according to standard protocols [28].

DNA fragment of the crp coding section was amplified from Streptomyces cinnamonensis genome DNA, using CZ-F and CZ-R as primers, and integrated into pLCY009 between Nde I and Xba I sites, generating the integrative $c r p$ overexpression vector pLCY009-crp. The nucleotide sequence of the $c r p$ gene was deposited under the GenBank accession number KY305484. 
Antisense RNA vector was constructed to hinder the translation of the crp gene. A 120-bp reverse complementary sequence to 60-bp 5' untranslation region (UTR), RBS and 60-bp coding sequence was designed as the binding sequence of the crp mRNA. This binding sequence was flanked with one restrict site and 21-bp inverted repeat overhangs to form a hairpin in the $5^{\prime}$ and $3^{\prime}$ ends [29]. This 174-bp DNA fragment containing binding part and hairpin part was amplified using primers CS1-F and CS1-R, and then was integrated into pLCY010 between Hind III and Xba I sites, generating the replicative anti-crp RNA vector pLCY010-csRNA.

Table 1. Bacterial strains and plasmids used in this study.

\begin{tabular}{|c|c|c|}
\hline Strains/Plasmids & Characteristics & Reference or Source \\
\hline \multicolumn{3}{|l|}{ Strains } \\
\hline \multicolumn{3}{|l|}{ Escherichia coli } \\
\hline ET12567/pUZ8002 & Strain for conjugal transfer, with pUZ8002 helper plasmids, $\mathrm{Chl}^{\mathrm{R}}, \mathrm{Kan}^{\mathrm{R}}$ & [28] \\
\hline \multicolumn{3}{|c|}{ Streptomyces cinnamonensis } \\
\hline ST021 & The parental strain derived from Streptomyces cinnamonensis ATCC15413 & This work \\
\hline \multicolumn{3}{|c|}{ - } \\
\hline pLCY009 & pIB139 derivative, $\Phi C 31$ attachment site, integrative, carrying $\mathrm{P}_{\text {ermE* }}$ promoter, $\mathrm{Hyg}^{\mathrm{R}}$ & [27] \\
\hline pLCY010 & pUWL201 derivative, pIJ101 replicon, replicative, carrying $\mathrm{P}_{\mathrm{ermE}^{*}}$ promoter, $\mathrm{Amp}^{\mathrm{R}}, \mathrm{Tsr}^{\mathrm{R}}, \mathrm{Hyg}^{\mathrm{R}}$ & [30] \\
\hline pLCY009-crp & crp overexpression vector, $\Phi C 31$ attachment site, integrative, carrying $\mathrm{P}_{\mathrm{ermE}^{*}}$ promoter, $\mathrm{Hyg}^{\mathrm{R}}$ & This work \\
\hline pLCY010-csRNA & Anti-crp RNA vector, pIJ101 replicon, replicative, carrying $\mathrm{P}_{\text {ermE}^{*}}$ promoter, $\mathrm{Hyg}^{\mathrm{R}}$ & This work \\
\hline
\end{tabular}

\subsection{Construction of Engineered S. cinnamonensis Strains}

The recombinant plasmids were transferred into $S$. cinnamonensis via conjugation with the help of E. coli ET12567. Overexpression strain ST042 and anti-crp strain ST043 were generated from strain ST021 introduced with pLCY009-crp and pLCY010-csRNA, respectively.

\subsection{RNA-Seq Library Preparation, Clustering and Sequencing}

RNA was extracted from 6-day mycelia in fermentation medium, and $3 \mu \mathrm{g}$ RNA was used to prepare each sample. NEBNext Ultra Directional RNA Library Prep Kit for Illumina (NEB, Ipswich, MA, USA) was used to generate sequencing libraries according to the manufacturer's protocol. The library quality was assessed with the Agilent Bioanalyzer 2100 system.

The clustering of the index-coded samples was performed on a cBot Cluster Generation System using TruSeq PE Cluster Kit v3-cBot-HS (Illumina, San Diego, CA, USA) following the manufacturer's recommendation. Illumina Hiseq platform was employed to sequence the library and generate paired-end reads. The transcriptome data of ST021, ST042 and ST043 have been deposited in the NCBI Sequence Read Archive (SPA) under accession number PRJNA398614.

\subsection{Quantification and Differential Analysis of Gene Expression}

The reads number mapped to each gene was counted by HTSeq v0.6.1. FPKM (expected number of Fragments Per Kilobase of transcript sequence per Million base pairs sequenced) is the most commonly used method for estimating gene expression levels [31], and it was calculated based on the length of each gene and read counts mapped to one gene.

The read counts of each library were adjusted by edgeR program package through one scaling normalized factor. DEGSeq $\mathrm{R}$ package was employed to analyze differential expression of different conditions [32]. The $p$-values were adjusted according to the Benjamini and Hochberg method. The $q$-value (adjusted $p$-value) of 0.005 and log2 (Fold change) of 1 were set as the threshold for significantly differential expression. 


\subsection{GO and KEGG Enrichment Analysis of Differentially Expressed Genes}

GOseq R package was used to analyze gene ontology (GO) enrichment of differentially expressed genes (DEGs). Gene length bias was corrected in the final results. GO terms with $q$-values under 0.05 were considered to be significantly enriched. Statistical enrichment of DEGs in KEGG pathways was tested by KOBAS software. Model Streptomyces coelicolor was chosen as the reference microorganism.

\subsection{Semi-Quantitative RT-PCR}

Primers involved in RT-PCR experiments are listed in Supplementary Table S1. RNA was isolated from the 6-day S. cinnamonensis mycelia in fermentation medium. Reverse transcription was conducted employing First-Strand cDNA Synthesis SuperMix (TransGen Biotech, Beijing, China) using $500 \mathrm{ng}$ of total RNA as template. The resultant cDNAs, diluted five times, were used as template in PCR analysis. PCR conditions were set as follows: $95^{\circ} \mathrm{C}$ for $5 \mathrm{~min} ; 30$ cycles of $95^{\circ} \mathrm{C}$ for $5 \mathrm{~s}, 55-60{ }^{\circ} \mathrm{C}$ for $30 \mathrm{~s}$ and $72{ }^{\circ} \mathrm{C}$ for $30 \mathrm{~s} ; 72{ }^{\circ} \mathrm{C}$ for $10 \mathrm{~min}$. PCR products were separated by agarose gel electrophoresis and exposed under UV to calculate their relative intensities by densitometric analysis software. All data were verified by three independent experiments.

\subsection{Extraction and Analysis of Monensin}

Monensin was extracted from mycelium cells and the titer was measured by HPLC [27]. In detail, mycelium cells of $S$. cinnamonensis were separated from fermentation broth after centrifugation, washed with PBS three times and resuspended in methanol. After ultrasonic cytolysis for $1 \mathrm{~h}$, the lysate was centrifuged and the supernatant was filtered with $0.22 \mu \mathrm{m}$ membrane and used to evaluate monensin titer. Analysis was carried out by HPLC using an Agela $5 \mu \mathrm{m}$ ODS3 $100 \AA$ A column $(250 \mathrm{~mm} \times 4.6 \mathrm{~mm})$, and the titer was quantified by Alltech ELSD 2000 evaporative light scattering detector. The mobile phase was composed of solvent $\mathrm{A}$ ( $20 \mathrm{mM}$ ammonium acetate) and solvent $\mathrm{B}$ (methanol), and the linear gradient program was set as follows: $80 \%-100 \%$ solvent B (0-25 min) and $100 \%-80 \%$ solvent B (25-30 $\mathrm{min})$. The flow rate was at a constant $1 \mathrm{~mL} / \mathrm{min}$.

\section{Results}

\subsection{Crp Positively Regulates Monensin Biosynthesis}

To evaluate the regulatory role of Crp in monensin biosynthesis, the crp overexpression strain ST042 and the anti-crp stain ST043 with anti-crp sequences (Figure 1a) were constructed, and monensin yield and mycelium dry weight (MDW) at intervals of two days throughout fermentation were detected. The mycelium growth was early stimulated by overexpressing the crp gene and retarded by silencing the $\operatorname{crp}$ gene (Figure $1 \mathrm{~b}$ ), implying that $\mathrm{Crp}$ has a positive effect on the vegetative development of $S$. cinnamonensis. Furthermore, compared to the original strain ST021 (14.9 mg monensin/mg MDW) (Figure 1c), strain ST042 produced $19.6 \mathrm{mg}$ monensin per mg MDW after 10-day fermentation with $31.5 \%$ increase, while strain ST043 produced $8.18 \mathrm{mg}$ monensin per mg MDW with $45.1 \%$ reduction. The results indicated that Crp positively regulates monensin biosynthesis, which was not due to the mycelium growth. 

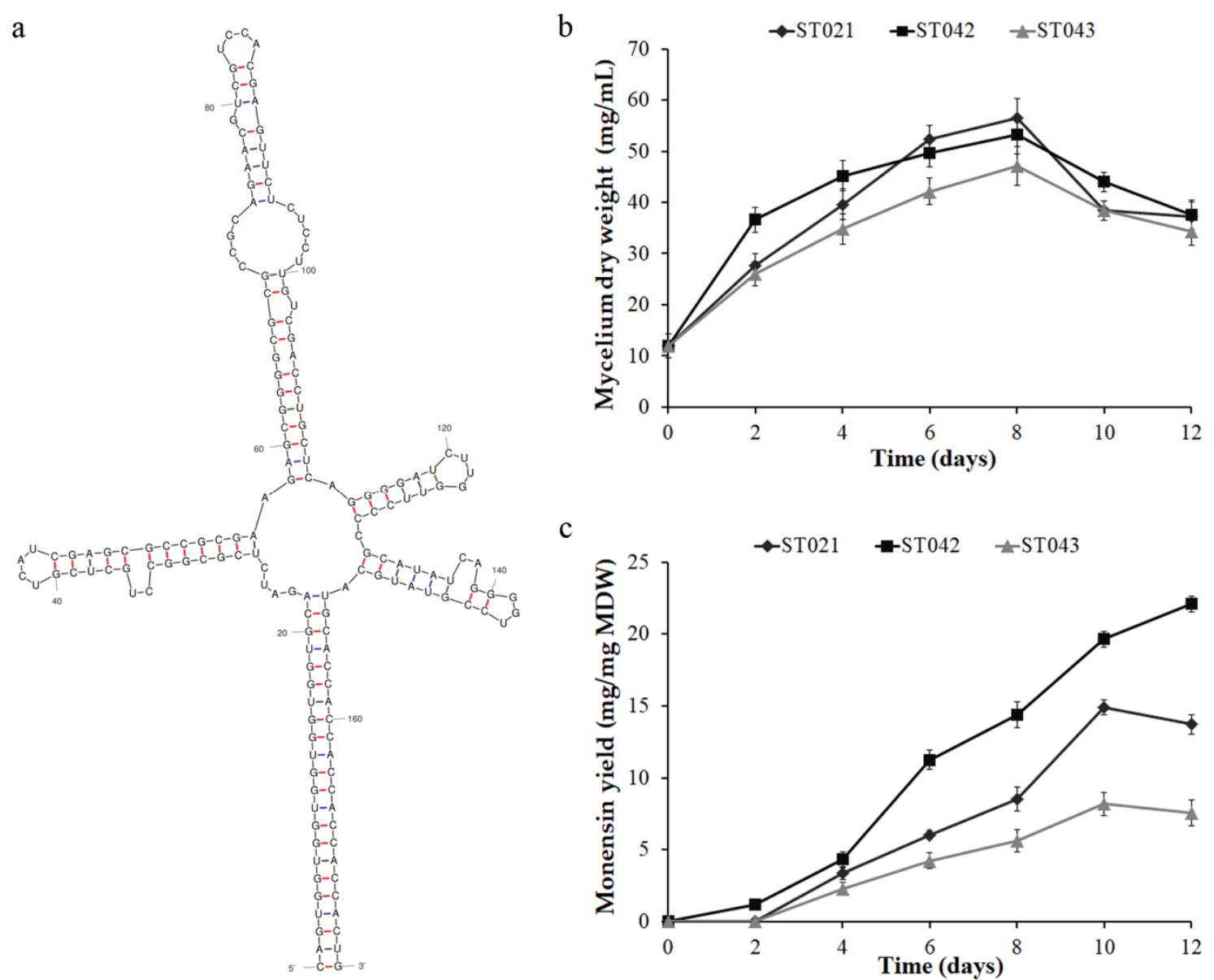

Figure 1. The overexpression and antisense RNA silencing of the crp gene. (a) The predicted secondary structure of anti-crp sequences on pLCY010-csRNA vector. Mycelium dry weight (MDW) (b) and monensin yield (c) of the original strain ST021, the crp overexpression strain ST042 and the anti-crp strain ST043 of S. cinnamonensis. Error bars indicate standard deviations from three independent experiments.

\subsection{RNA-Seq Shows Global Regulatory Roles of Crp}

To gain insight into the regulatory mechanism of Crp on monensin biosynthesis, RNA sequencing was conducted. Totals of 15.7, 20.6 and 22.7 million clean reads from strain libraries of ST021, ST042 and ST043 were obtained after filtration of 16.5, 21.2 and 23.3 million raw reads, respectively, responding for more than $93.94 \%$ at the Q20 level (sequence error probability of $0.04 \%$ ). These clean reads were mapped to the S. cinnamonensis ST021 genome, resulting in $15.6(99.29 \%), 17.9(86.75 \%)$ and 20.7 $(91.16 \%)$ million mapped reads for ST021, ST042 and ST043, respectively (Table 2).

Table 2. Statistical analysis of RNA sequence data and genome mapping.

\begin{tabular}{cccc}
\hline Samples & ST021 & ST042 & ST043 \\
\hline Raw reads & $16,537,074$ & $21,162,804$ & $23,277,776$ \\
Clean reads & $15,709,834$ & $20,599,352$ & $22,714,616$ \\
Clean bases (G) & 1.96 & 2.57 & 2.84 \\
GC content (\%) & 65.57 & 63.56 & 66.01 \\
Q20 Base ratio (\%) & 93.94 & 97.27 & 97.26 \\
Q30 Base ratio (\%) & 88.06 & 93.11 & 93.05 \\
Mapped reads & $15,597,581$ & $17,869,047$ & $20,707,208$ \\
Mapped reads ratio (\%) & 99.29 & 86.75 & 91.16 \\
\hline
\end{tabular}

* Q20 Base ratio: error probability of 1\%. Q30 Base ratio: error probability of $0.1 \%$. 
The levels of gene expression were evaluated by FPKM values. On the basis of the applied criteria ( $q$-value $<0.005$ and fold changes $\geq 2$ ), DEGs were identified among strains ST021, ST042 and ST043. When compared to the original strain ST021, 756 genes were upregulated and 645 genes were downregulated in the crp overexpression strain ST042 (Figure 2a), while 1237 genes were upregulated and 1326 genes were downregulated in the anti-crp strain ST043 (Figure 2b). Additionally, strains ST042 and ST043 shared 820 DGEs compared to ST021 (Figure 2c). Particularly, the expression levels of the $c r p$ gene in three strains were evaluated. The FPKM values of the crp gene were 418.32 and 116.51, in the crp overexpression strain ST042 and the anti-crp strain ST043, representing 2.2-fold enhancement and $38.3 \%$ decrease, respectively, compared to the original strain ST021, in which FPKM value of the crp gene was 188.91. These results suggested the extensive effects of Crp on the regulation of gene expression in S. cinnamonensis. A total of 3144 genes were differentially expressed under the conditions of the overexpression and partial silencing of the crp gene.

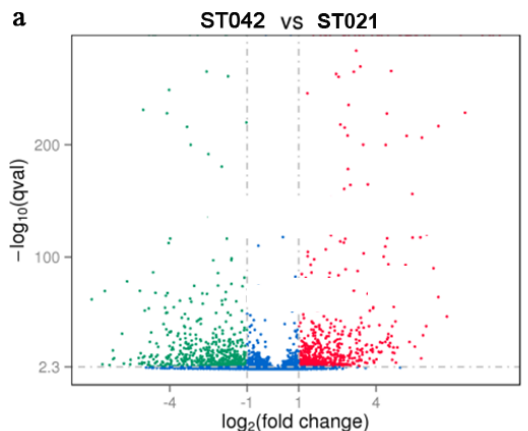

b

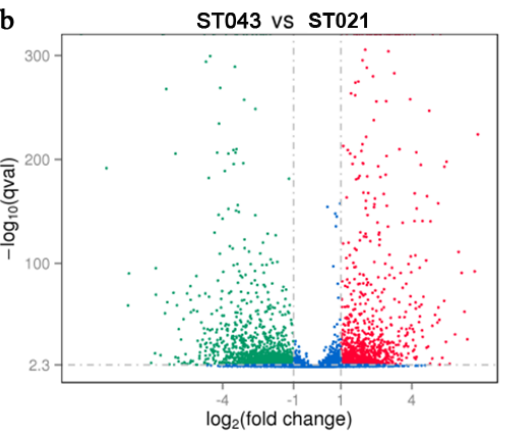

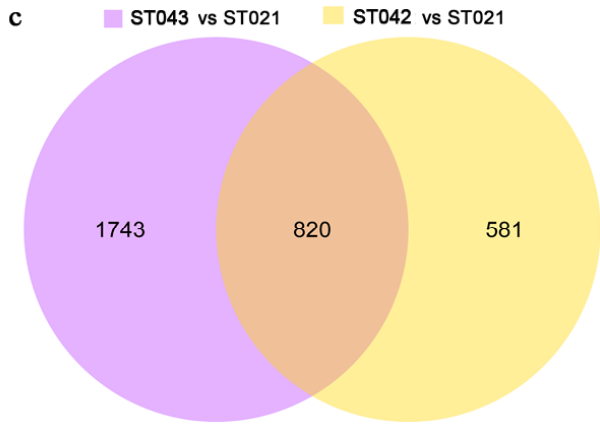

Figure 2. The volcano plots and Venn diagram of differential expressed genes (DEGs) in strains ST021, ST042 and ST043. Volcano plots of ST042 vs. ST021 (a) and ST043 vs. ST021 (b). DEGs are shown in red (upregulated) and green (downregulated) points, while the others are shown in blue points. The horizontal axis represents the fold change of gene expression in different samples, while the vertical axis represents the statistical significance of gene expression difference. (c) Venn diagram of DEGs. Compared with ST021, the number of DEGs in ST043 is shown in the purple circle on the left, while that in ST042 is in orange circle on the right. The intersection of the two circles represents the number of DEGs shared by ST042 and ST043.

To investigate the functions of DEGs influenced by Crp, gene ontology (GO) enrichment was used for describing the biological roles of genes and their coding proteins [33]. All genes were classified into function groups in three main categories (Figure 3). In the biological process category, gene expression (GO: 0010467), cellular protein metabolic process (GO: 0044267) and translation (GO: 0006412) were significantly enriched. Nine GO terms including intracellular part (GO: 0044424), cytoplasm (GO: 0005737) and organelle (GO: 0043226) were significantly enriched in the cellular component category. In the molecular function category, structural molecule activity (GO: 0005198), RNA binding (GO: 0003723) and structural constituent of ribosome (GO: 0003735) were significantly enriched. These 
results further revealed the broad regulatory effects of Crp on various intracellular functions in $S$. cinnamonensis.

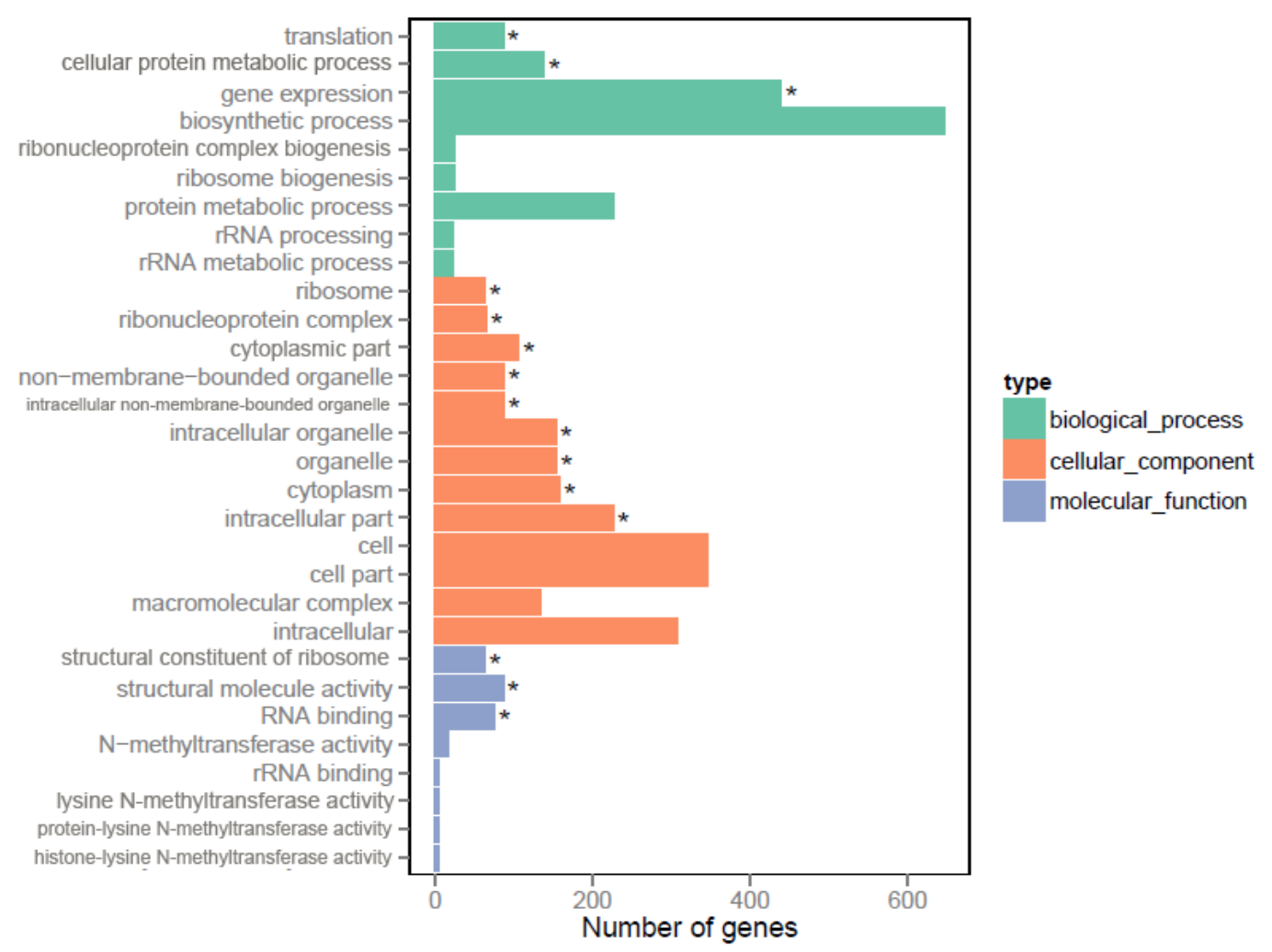

Figure 3. Functional categorization of DEGs in gene ontology (GO) in strain ST042. The asterisks indicate significantly enriched GO terms.

\subsection{Crp Upregulates Biosynthetic Pathways of Monensin Precursors}

Malonyl-CoA, methylmalonyl-CoA and ethylmalonyl-CoA are precursors for monensin biosynthesis and are derived from primary metabolism. To decipher the genes involved in the precursor biosynthesis, the 777 DEGs were mapped to the 92 KEGG pathways in the database. The majority of the 57 DEGs involved in glycolysis, fatty acid degradation and other carbon metabolisms (Table 3, Table S2) were transcriptionally promoted by the crp overexpression in strain ST042 and inhibited by the crp partial silencing in strain ST043 (Figure 4a). The 10 DEGs enriched in glycolysis were responsible for acetyl-CoA formation from glucose via pyruvate metabolism and consequently in increasing the biosynthesis of malonyl-CoA. In fatty acid degradation, most of the 20 DEGs code acyl-CoA dehydrogenases, acetyl-CoA acetyltransferases and fatty-acid-CoA ligases. In carbon metabolism, the 27 DEGs are involved in the biosynthesis of butyryl-CoA and ethylmalonyl-CoA from crotonyl-CoA and of methylmalonyl-CoA from the intermediate succiny-CoA of the TCA cycle, which would enhance the supply of acyl-CoA building blocks in monensin biosynthesis.

Table 3. Selected KEGG enrichment results of DEGs between strains ST042 and ST021.

\begin{tabular}{cccc}
\hline Pathway ID & Pathway & Number of DEGs & Representative Genes * \\
\hline sco00010 & Glycolysis & 10 & acs, $p c k A$ \\
sco00071 & Fatty acid degradation & 20 & $a c d H$, atoB \\
sco01200 & Carbon metabolism & 27 & accB, $m u t B$, epi, ccr \\
\hline \multicolumn{4}{r}{ * The putative functions of genes involved are listed in Supplementary Table S2. }
\end{tabular}



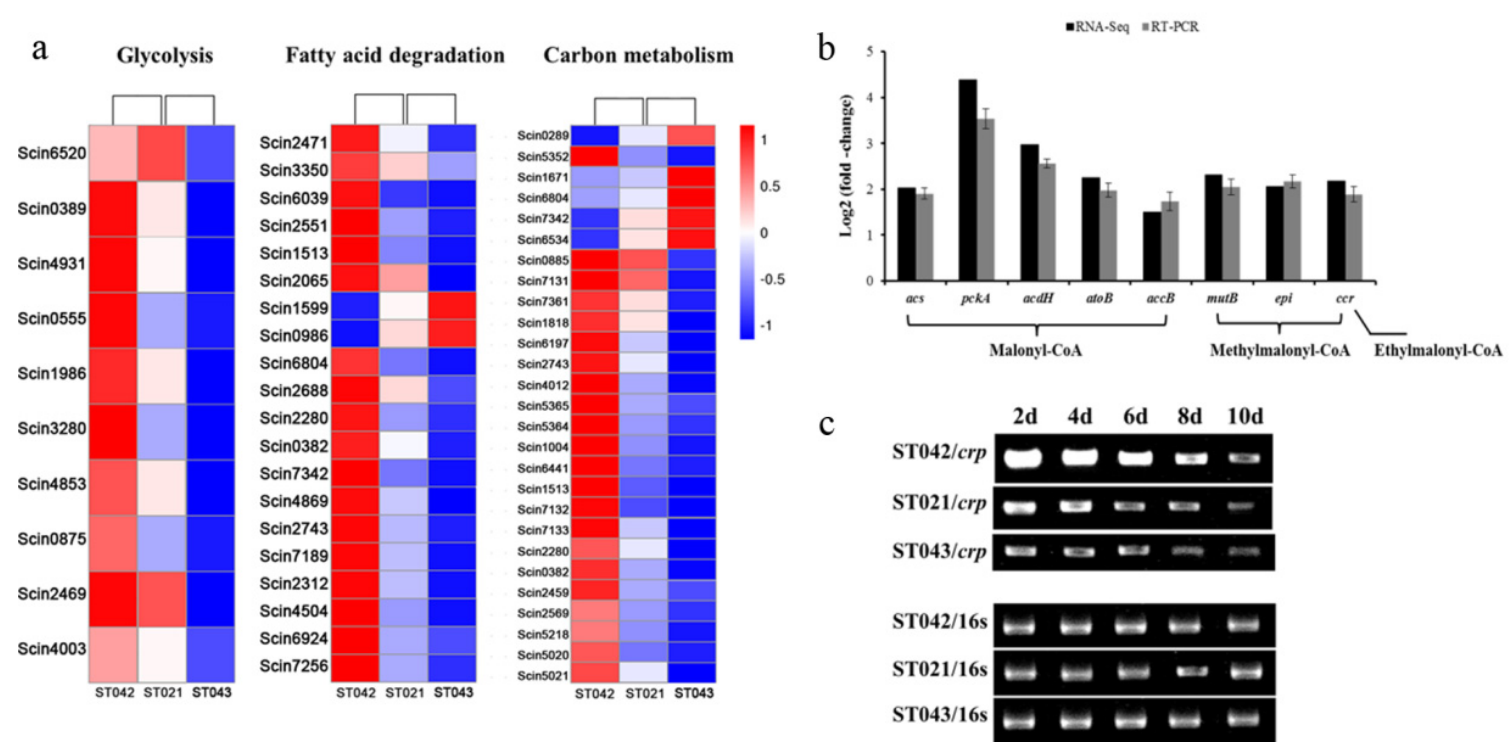

Figure 4. Transcription analysis of selected primary metabolic genes. (a) Heat maps of 57 DEGs enriched in the processes of glycolysis (10), fatty acid degradation (20) and carbon metabolism (27). Gene names involved are listed beside each heat map. (b) The relative transcriptional levels of primary metabolic genes in precursor biosynthesis in the crp overexpression strain ST042 by RNA-Seq and RT-PCR. Those in the original strain ST021 were set to 1.0 (arbitrary units). acs, acetyl-CoA synthetase gene; $p c k A$, phosphoenolpyruvate carboxykinase gene; $a c d H$, acyl-CoA dehydrogenase gene; atoB, acetyl-CoA acetyltransferase gene; $a c c B$, acyl-CoA carboxylase complex B subunit gene; $m u t B$, coenzyme B12-dependent methylmalonyl-CoA mutase B subunit gene; epi, methylmalonyl-CoA epimerase gene; $c c r$, crotonyl-CoA reductase gene. Error bars indicate standard deviations from three independent experiments. (c) The crp transcription of ST042, ST021 and ST043 at different intervals.

In order to identify the DEGs responding for biosynthesis of precursors malonyl-CoA, methylmalonyl-CoA and ethylmalonyl-CoA, several representative genes in those metabolic pathways were chosen and reverse-transcription PCR was performed with specific primers listed in Table S1. Compared to the original strain ST021, the transcriptional levels of acs (Scin0389), pckA (Scin4931), $a c d H$ (Scin4869), atoB (Scin7189) and $a c c B$ (Scin4012) genes in malonyl-CoA biosynthetic pathways and mutB (Scin5352), epi (Scin6197) and ccr (Scin6441) genes in methylmalonyl-/ethylmalonyl-CoA biosynthetic pathways were increased 1.63-fold to 3.34-fold in strain ST042 (Figure 4b) and decreased by $32 \%$ to $65 \%$ in strain ST043. The results of RT-PCR analysis were comparable to RNA-Seq data (Figure 4b), confirming that Crp enhanced monensin biosynthesis via upregulating biosynthesis of the short chain acyl-CoA precursors. In addition, time-course transcription analysis of the crp gene in strains ST021, ST042 and ST043 was conducted. At different intervals in the fermentation course, the transcriptional levels of the crp gene were constantly higher in ST042 and lower in ST043 than those in ST021, which was consistent with the results of RNA sequencing (Figure 4c).

\subsection{Crp Upregulates Transcription of mon Cluster}

There are twenty genes in the mon cluster, and our previous study suggested that the monensin biosynthetic gene cluster is transcribed into eight transcription units (TUs) [26], shown in Figure 5a. The RNA-seq analysis results showed that TU4, TU5, TU6, TU7 and TU8 in the mon cluster were significantly upregulated in the crp overexpression strain ST042 and correspondingly downregulated in the anti-crp strain ST043, compared to the original strain ST021 (Figure 5b). In contrast, TU2 was downregulated in ST042 and ST043. The transcription of TU1 was decreased in ST043 and not obviously changed in ST042, while the transcription of TU3 was increased in ST042 and not obviously changed in ST043. Thus, RT-PCR was further conducted and the transcripts of mon TUs were assessed. When crp was overexpressed, the transcripts of TU4, TU5, TU6, TU7 and TU8 were increased by 2.1-fold to 
4.8-fold compared to the original strain ST021. In contrast, partial crp silencing resulted in $34.0 \%$ to $71 \%$ decrease of transcripts of these five TUs (Figure $5 \mathrm{c}$ ). No significant changes in the transcripts of TU1, TU2 and TU3 were detected by RT-PCR whenever the crp gene was overexpressed or partially inhibited (Figure 5c). Taken together, Crp positively upregulated the transcription of five mon TUs, including PKS genes, tailoring genes and pathway-specific regulatory gene monRI.

a

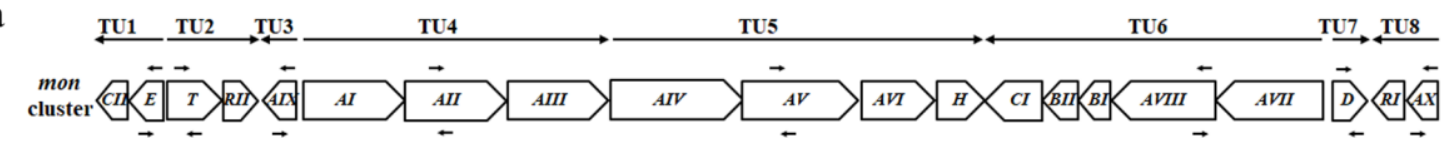

b

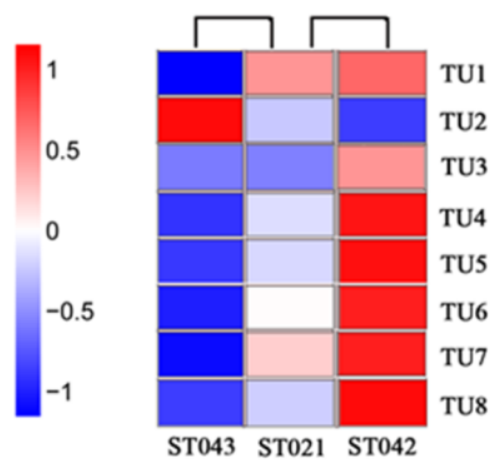

c

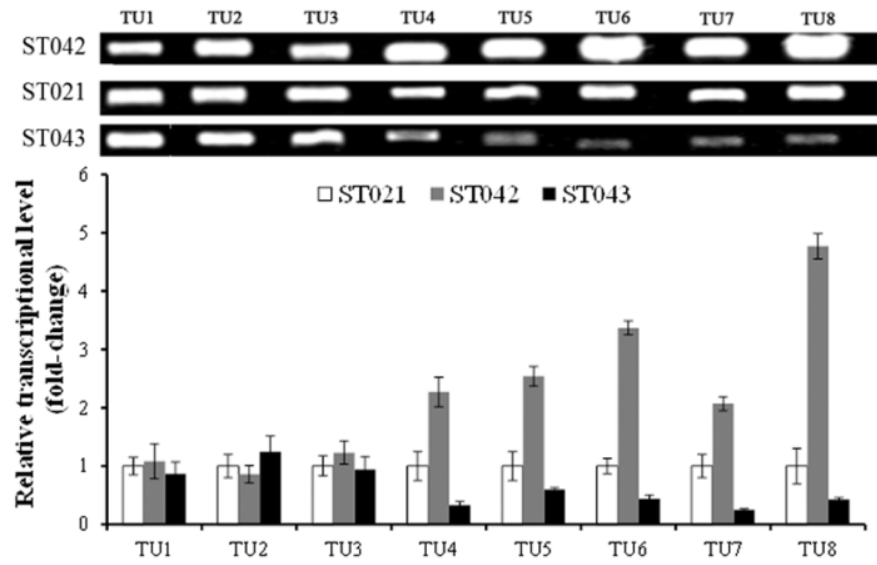

Figure 5. RNA-Seq analysis and RT-PCR verification for transcription of mon cluster. (a) Transcription units (TUs) in monensin biosynthesis. (b) Heat map of clustering analysis of eight mon TUs by RNA-seq. Expression ratios are shown as $\log 2$ values. Red color represents increased expression, while blue color represents decreased expression. (c) Relative transcriptional levels of TUs in strains ST042 (grey column) and ST043 (black column) compared to strain ST021 (white column), which were set to 1.0 (arbitrary units) by RT-PCR. Error bars indicate standard deviations from three independent experiments.

\section{Discussion}

In this work, the forward and reverse genetic experiments were conducted, the differential gene expressions were analyzed by RNA sequencing and RT-PCR and the global positive regulation of Crp on monensin biosynthesis in S. cinnamonensis was deciphered. Crp enhanced the expression of the crucial genes involved in the biosynthesis of acetyl-CoA, malonyl-CoA, methylmalonyl-CoA and ethylmalonyl-CoA. In the crp overexpression strain ST042, the DEGs enriched in glycolysis were mainly responsible for glucose utilization and pyruvate metabolism, which would consequently increase the supply of acetyl-CoA. In fatty acid degradation, the majority of the DEGs coded acyl-CoA dehydrogenases, which were involved in each step of long-chain fatty acid degradation. Furthermore, genes that coded acetyl-CoA acetyltransferases and long chain fatty acid CoA ligases were also transcriptionally promoted by the crp overexpression. In carbon metabolism, the products of the DEGs were involved in a variety of reactions, including the synthesis of malonyl-CoA from acetyl-CoA, the metabolism of the intermediate succiny-CoA in TCA cycle to methylmalonyl-CoA and the conversion of crotonyl-CoA to butyryl-CoA and ethylmalonyl-CoA, which would enhance the supply of acyl-CoA building blocks in monensin biosynthesis. Furthermore, the majority of genes in the mon cluster were also upregulated by Crp.

The sufficient availability of precursors, which are supplied from primary metabolism, is a prerequisite for the biosynthesis and production of secondary metabolites [34]. In monensin biosynthesis, five malonyl-CoA, seven methylmalonyl-CoA and one ethylmalonyl-CoA were proposed to be loaded by thirteen acetyltransferase (AT) domains of mon PKSs and used as extender units in the carbon backbone formation of one monensin A molecule [19,35,36]. Acetyl-CoA is the central carbon 
node connecting precursor supply from primary pathways with monensin biosynthesis. In oil-rich fermentation medium, the fatty acid degradation pathway can provide acetyl-CoA for monensin biosynthesis [37]. Additionally, when glucose is utilized for mycelium growth, acetate is the major byproduct, and acetyl-CoA synthetase (ACS) is responsible for the synthesis of acetyl-CoA from acetate. The corresponding genes of acs in the glycolytic pathway and acdH (coding acyl-CoA dehydrogenase) and $a t o B$ (coding acetyl-CoA acetyltransferase) in the fatty acid degradation pathway were upregulated by Crp, indicating that the formation of acetyl-CoA is enhanced to improve monensin production in $S$. cinnamonensis.

The canonical extender unit of polyketide biosynthesis, malonyl-CoA, is mainly derived from carboxylation of acetyl-CoA by acetyl-CoA carboxylases (ACC) [38,39]. Overexpression of acc genes led to significant increase in the production of polyketides actinorhodin in S. coelicolor [40] and mithramycin in S. argillaceus [41]. When the crp gene was overexpressed in S. cinnamonensis, the transcription of the $a c c B$ gene was upregulated, which would increase malonyl-CoA supply for monensin biosynthesis.

As seven methylmalonyl-CoA extender units are required to build a single polyketide of monensin, the supply of methylmalonyl-CoA may represent a limiting step in the biosynthesis of monensin [36]. Succinyl-CoA stemming from the TCA cycle was sequentially catalyzed by methylmalonyl-CoA mutase (Mut) and methylmalonyl-CoA epimerase (Epi) to (2R)- then to (2S)-methylmalonyl-CoA [42]. Phosphoenolpyruvate is converted into oxaloacetate by phosphoenolpyruvate carboxykinase (PckA), which allows carbon flux from glucose toward the TCA cycle to strengthen the formation of succinyl-CoA. Additionally, crotonyl-CoA reductase (CCR) was proved to be essential for providing the majority of methylmalonyl-CoA for monensin biosynthesis and when S. cinnamonensis was grown on an oil-based medium $[37,43]$. Acetyl-CoA is orderly transformed into acetoacetyl-CoA by AtoB then into crotonyl-CoA, which will be subsequently catalyzed to butyryl-CoA by CCR. Butyryl-CoA pool can be used to generate not only ethylmalonyl-CoA but also methylmalonyl-CoA precursor pool under oil-based nutrient condition [37]. The transcription of $p c k A$, mutB, epi and $c c r$ were upregulated when the crp gene was overexpressed in strain ST042, which would result in more efficient biosynthesis of methylmalonyl-CoA and higher monensin titer.

Most importantly, Crp upregulates five transcription units in the mon cluster, which cover PKSs genes, tailoring genes and pathway-specific regulatory gene. In S. coelicolor, Crp regulated 8 out of 22 predicted secondary metabolic clusters, and its regulatory targets were composed of PKSs and tailoring genes in act, red, $c d a$ and ycpk clusters [12]. The monAI-monAVIII genes coding the PKSs are responsible for assembling precursors into the monensin backbone [44]. Then, tailoring enzyme MonCI mediates an epoxidation of the polyolefin constructed by polyketide synthases, and MonBI and MonBII cooperatively catalyze the cyclization of the resultant polyepoxide to yield the polyether skeleton of monensin. Our previous studies showed that three pathway-specific regulators (MonRI, MonRII and MonH) [26] and the global regulator DasR [27] do not regulate the transcriptions of mon PKSs genes and tailoring genes monCI, monBI and monBII. Here, our results suggested that the targets of Crp regulation would be the PKSs and monCI, monBI and monBII genes of the mon cluster. Moreover, Crp regulations on pathway-specific regulatory genes actII-ORF4, redZ and cdaR were identified in $S$. coelicolor [12]. Of three pathway-specific regulators for monensin biosynthesis, MonRI and MonRII upregulated the transcription of tailoring gene monD and thioesterase gene monAX [26], while monRI was under the control of Crp, indicating that Crp possibly exerted regulatory effects on the transcription of monD and monAX through its tuning on monRI transcription.

\section{Conclusions}

A global regulatory model of Crp on monensin biosynthesis in S. cinnamonensis was proposed in this study (Figure 6). Crp upregulated curial primary carbon metabolic genes involved in glycolysis ( $p c k A, a c s)$ and fatty acid degradation $(a t o B, a c d H)$, together with the genes involved in biosynthesis of malonyl-CoA $(a c c B)$, methylmalonyl-CoA (mutB, epi and $c c r)$ and ethylmalonyl-CoA (ccr), which might make great contribution to precursor supply in monensin biosynthesis. Crp also upregulated 
the transcription of mon cluster in multiple patterns, including enhancing the expression of PKSs genes, tailoring genes (monBI-monBII-monCI, monD and monAX) and a pathway-specific activator gene monRI. Our work sheds new light on the regulatory mechanism of Crp on monensin biosynthesis and extends the understanding of Crp functions in Streptomyces.

a. Regulation on precursors supply

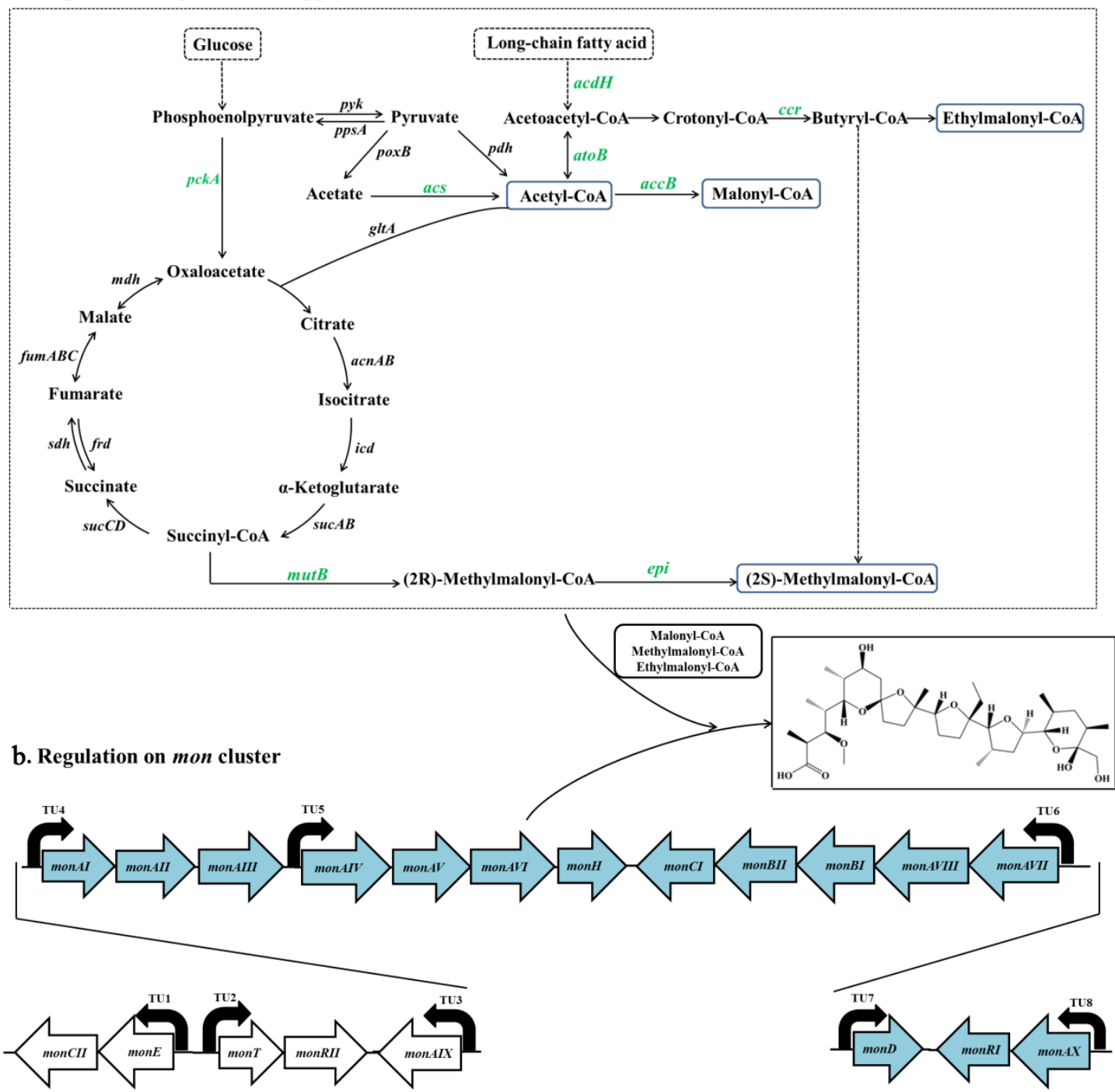

Figure 6. Proposed regulatory mechanism of Crp for monensin biosynthesis. (a) The primary metabolism of precursor supply for monensin biosynthesis. The metabolic genes under Crp regulation are shown in green. (b) The monensin biosynthetic gene cluster. The mon genes of the transcription units (TUs) under Crp regulation are shown in blue. Curved black arrows represent transcription directions. $p y k$, pyruvate kinase gene; $p p s A$, phosphoenolpyruvate synthase gene; $p o x B$, pyruvate oxidase gene; $p d h$, pyruvate dehydrogenase gene; $a c n A B$, aconitate hydratase gene; $i c d$, isocitrate dehydrogenase gene; sucABCD, succinyl CoA synthase gene; frd, fumarate reductase gene; $s d h$, succinate dehydrogenase gene; fum $A B C$, fumarase $A B C$ gene; $m d h$, malate dehydrogenase gene; glt $A$, citrate synthase gene.

Supplementary Materials: The following are available online at http://www.mdpi.com/2076-2607/8/2/271/s1, Table S1: Primers used in this study, Table S2: Putative gene functions of 57 DEGs.

Author Contributions: Conceptualization, G.-R.Z.; methodology, C.-Y.L. and Y.Z.; validation, J.-H.W. and R.-H.X.; formal analysis Y.Z.; data curation, Y.Z.; writing-original draft preparation, Y.Z.; writing-review and editing, C.-Y.L., G.-R.Z., and J.Q.; project administration, G.-R.Z. All authors have read and agreed to the published version of the manuscript. 
Funding: This research was supported by the National Key R\&D Program of China (2017YFD0201400) and the National Natural Science Foundation of China (grant No. 31370092).

Conflicts of Interest: The authors declare no conflict of interest.

\section{References}

1. Barka, E.A.; Vatsa, P.; Sanchez, L.; Gaveau-Vaillant, N.; Jacquard, C.; Klenk, H.-P.; Clément, C.; Ouhdouch, Y.; van Wezel, G.P. Taxonomy, physiology, and natural products of Actinobacteria. Microbiol. Mol. Biol. Rev. 2016, 80, 1-43. [CrossRef]

2. Liu, G.; Chater, K.F.; Chandra, G.; Niu, G.; Tan, H. Molecular regulation of antibiotic biosynthesis in Streptomyces. Microbiol. Mol. Biol. Rev. 2013, 77, 112-143. [CrossRef]

3. Baltz, R.H. Genetic manipulation of secondary metabolite biosynthesis for improved production in Streptomyces and other actinomycetes. J. Ind. Microbiol. Biotechnol. 2016, 43, 343-370. [CrossRef]

4. Urem, M.; Świątek-Połatyńska, M.A.; Rigali, S.; van Wezel, G.P. Intertwining nutrient-sensory networks and the control of antibiotic production in Streptomyces. Mol. Microbiol. 2016, 102, 183-195. [CrossRef]

5. Körner, H.; Sofia, H.J.; Zumft, W.G. Phylogeny of the bacterial superfamily of Crp-Fnr transcription regulators: Exploiting the metabolic spectrum by controlling alternative gene programs. FEMS Microbiol. Lett. 2003, 27, 559-592. [CrossRef]

6. Görke, B.; Stülke, J. Carbon catabolite repression in bacteria: Many ways to make the most out of nutrients. Nat. Rev. Microbiol. 2008, 6, 613-624. [CrossRef]

7. Nosho, K.; Fukushima, H.; Asai, T.; Nishio, M.; Takamaru, R.; Kobayashi-Kirschvink, K.J.; Ogawa, T.; Hidaka, M.; Masaki, H. cAMP-CRP acts as a key regulator for the viable but non-culturable state in Escherichia coli. Microbiology 2018, 164, 410-419. [CrossRef] [PubMed]

8. Grasso, L.L.; Martino, D.C.; Alduina, R. Production of antibacterial compounds from Actinomycetes. In Actinobacteria-Basics and Biotechnological Applications; IntechOpen: London, UK, 2016; pp. 177-198.

9. Derouaux, A.; Dehareng, D.; Lecocq, E.; Halici, S.; Nothaft, H.; Giannotta, F.; Moutzourelis, G.; Dusart, J.; Devreese, B.; Titgemeyer, F. Crp of Streptomyces coelicolor is the third transcription factor of the large CRP-FNR superfamily able to bind cAMP. Biochem. Biophys. Res. Commun. 2004, 325, 983-990. [CrossRef] [PubMed]

10. Derouaux, A.; Halici, S.; Nothaft, H.; Neutelings, T.; Moutzourelis, G.; Dusart, J.; Titgemeyer, F.; Rigali, S. Deletion of a cyclic AMP receptor protein homologue diminishes germination and affects morphological development of Streptomyces coelicolor. J. Bacteriol. 2004, 186, 1893-1897. [CrossRef] [PubMed]

11. Piette, A.; Derouaux, A.; Gerkens, P.; Noens, E.E.; Mazzucchelli, G.; Vion, S.; Koerten, H.K.; Titgemeyer, F.; De Pauw, E.; Leprince, P. From dormant to germinating spores of Streptomyces coelicolor A3 (2): New perspectives from the crp null mutant. J. Proteome Res. 2005, 4, 1699-1708. [CrossRef]

12. Gao, C.; Hindra; Mulder, D.; Yin, C.; Elliot, M.A. Crp is a global regulator of antibiotic production in streptomyces. mBio 2012, 3. [CrossRef] [PubMed]

13. Liao, C.H.; Yao, L.l.; Ye, B.C. Three genes encoding citrate synthases in Saccharopolyspora erythraea are regulated by the global nutrient-sensing regulators GlnR, DasR, and CRP. Mol. Microbiol. 2014, 94, 1065-1084. [CrossRef]

14. Chapman, H.D.; Jeffers, T.K.; Williams, R.B. Forty years of monensin for the control of coccidiosis in poultry. Poult. Sci. 2010, 89, 1788-1801. [CrossRef]

15. Ketola, K.; Vainio, P.; Fey, V.; Kallioniemi, O.; Iljin, K. Monensin is a potent inducer of oxidative stress and inhibitor of androgen signaling leading to apoptosis in prostate cancer cells. Mol. Cancer Ther. 2010, 9, 3175-3185. [CrossRef] [PubMed]

16. Tumova, L.; Pombinho, A.R.; Vojtechova, M.; Stancikova, J.; Gradl, D.; Krausova, M.; Sloncova, E.; Horazna, M.; Kriz, V.; Machonova, O. Monensin inhibits canonical Wnt signaling in human colorectal cancer cells and suppresses tumor growth in multiple intestinal neoplasia mice. Mol. Cancer Ther. 2014, 13, 812-822. [CrossRef] [PubMed]

17. Deng, Y.; Zhang, J.; Wang, Z.; Yan, Z.; Qiao, M.; Ye, J.; Wei, Q.; Wang, J.; Wang, X.; Zhao, L.; et al. Antibiotic monensin synergizes with EGFR inhibitors and oxaliplatin to suppress the proliferation of human ovarian cancer cells. Sci. Rep. 2015, 5, 17523. [CrossRef] 
18. Leadlay, P.; Staunton, J.; Oliynyk, M.; Bisang, C.; Cortes, J.; Frost, E.; Hughes-Thomas, Z.; Jones, M.; Kendrew, S.; Lester, J. Engineering of complex polyketide biosynthesis-insights from sequencing of the monensin biosynthetic gene cluster. J. Ind. Microbiol. Biotechnol. 2001, 27, 360-367. [CrossRef]

19. Oliynyk, M.; Stark, C.B.; Bhatt, A.; Jones, M.A.; Hughes-Thomas, Z.A.; Wilkinson, C.; Oliynyk, Z.; Demydchuk, Y.; Staunton, J.; Leadlay, P.F. Analysis of the biosynthetic gene cluster for the polyether antibiotic monensin in Streptomyces cinnamonensis and evidence for the role of monB and monC genes in oxidative cyclization. Mol. Microbiol. 2003, 49, 1179-1190. [CrossRef]

20. Risdian, C.; Mozef, T.; Wink, J. Biosynthesis of polyketides in Streptomyces. Microorganisms. 2019, 7, 124. [CrossRef]

21. Gallimore, A.R.; Stark, C.B.; Bhatt, A.; Harvey, B.M.; Demydchuk, Y.; Bolanos-Garcia, V.; Fowler, D.J.; Staunton, J.; Leadlay, P.F.; Spencer, J.B. Evidence for the role of the monB genes in polyether ring formation during monensin biosynthesis. Chem. Biol. 2006, 13, 453-460. [CrossRef]

22. Sato, K.; Minami, A.; Ose, T.; Oguri, H.; Oikawa, H. Remarkable synergistic effect between MonBI and MonBII on epoxide opening reaction in ionophore polyether monensin biosynthesis. Tetrahedron Lett. 2011, 52, 5277-5280. [CrossRef]

23. Minami, A.; Ose, T.; Sato, K.; Oikawa, A.; Kuroki, K.; Maenaka, K.; Oguri, H.; Oikawa, H. Allosteric regulation of epoxide opening cascades by a pair of epoxide hydrolases in monensin biosynthesis. ACS Synth. Biol. 2013, 9, 562-569. [CrossRef] [PubMed]

24. Hüttel, W.; Spencer, J.B.; Leadlay, P.F. Intermediates in monensin biosynthesis: A late step in biosynthesis of the polyether ionophore monensin is crucial for the integrity of cation binding. Beilstein. J. Org. Chem. 2014, 10, 361-368. [CrossRef] [PubMed]

25. Harvey, B.M.; Hong, H.; Jones, M.A.; Hughes-Thomas, Z.A.; Goss, R.M.; Heathcote, M.L.; Bolanos-Garcia, V.M.; Kroutil, W.; Staunton, J.; Leadlay, P.F. Evidence that a novel thioesterase is responsible for polyketide chain release during biosynthesis of the polyether ionophore monensin. ChemBioChem. 2006, 7, 1435-1442. [CrossRef] [PubMed]

26. Tang, Z.-K.; Li, X.-M.; Pang, A.-P.; Lin, C.-Y.; Zhang, Y.; Zhang, J.; Qiao, J.; Zhao, G.-R. Characterization of three pathway-specific regulators for high production of monensin in Streptomyces cinnamonensis. Appl. Microbiol. Biotechnol. 2017, 101, 6083-6097. [CrossRef] [PubMed]

27. Zhang, Y.; Lin, C.-Y.; Li, X.-M.; Tang, Z.-K.; Qiao, J.; Zhao, G.-R. DasR positively controls monensin production at two-level regulation in Streptomyces cinnamonensis. J. Ind. Microbiol. Biotechnol. 2016, 43, 1681-1692. [CrossRef]

28. Kieser, T.; Bibb, M.J.; Buttner, M.J.; Chater, K.F.; Hopwood, D.A. Practical streptomyces genetics; John Innes Foundation Norwich: Norwich, UK, 2000.

29. Uguru, G.C.; Mondhe, M.; Goh, S.; Hesketh, A.; Bibb, M.J.; Good, L.; Stach, J.E. Synthetic RNA silencing of actinorhodin biosynthesis in Streptomyces coelicolor A3 (2). PLoS ONE 2013, 8, e67509. [CrossRef]

30. Pang, A.-P.; Du, L.; Lin, C.-Y.; Qiao, J.; Zhao, G.-R. Co-overexpression of $l m b W$ and metK led to increased lincomycin A production and decreased byproduct lincomycin B content in an industrial strain of Streptomyces lincolnensis. J. Appl. Microbiol. 2015, 119, 1064-1074. [CrossRef]

31. Trapnell, C.; Williams, B.A.; Pertea, G.; Mortazavi, A.; Kwan, G.; Van Baren, M.J.; Salzberg, S.L.; Wold, B.J.; Pachter, L. Transcript assembly and quantification by RNA-Seq reveals unannotated transcripts and isoform switching during cell differentiation. Nat. Biotechnol. 2010, 28, 511-515. [CrossRef]

32. Wang, L.; Feng, Z.; Wang, X.; Wang, X.; Zhang, X. DEGseq: An R package for identifying differentially expressed genes from RNA-seq data. Bioinformatics. 2009, 26, 136-138. [CrossRef]

33. Young, M.D.; Wakefield, M.J.; Smyth, G.K.; Oshlack, A. Gene ontology analysis for RNA-seq: Accounting for selection bias. Genome. Biol. 2010, 11, R14. [CrossRef] [PubMed]

34. Bilyk, O.; Luzhetskyy, A. Metabolic engineering of natural product biosynthesis in actinobacteria. Curr. Opin. Biotechnol. 2016, 42, 98-107. [CrossRef] [PubMed]

35. Haydock, S.F.; Aparicio, J.F.; Molnár, I.; Schwecke, T.; Khaw, L.E.; König, A.; Marsden, A.F.; Galloway, I.S.; Staunton, J.; Leadlay, P.F. Divergent sequence motifs correlated with the substrate specificity of (methyl) malonyl-CoA: Acyl carrier protein transacylase domains in modular polyketide synthases. FEBS Lett. 1995, 374, 246-248. [CrossRef] 
36. Zhang, W.; Reynolds, K.A. MeaA, a putative coenzyme B12-dependent mutase, provides methylmalonyl coenzyme A for monensin biosynthesis in Streptomyces cinnamonensis. J. Bacteriol. 2001, 183, 2071-2080. [CrossRef]

37. Li, C.; Florova, G.; Akopiants, K.; Reynolds, K.A. Crotonyl-coenzyme A reductase provides methylmalonyl-CoA precursors for monensin biosynthesis by Streptomyces cinnamonensis in an oil-based extended fermentation. Microbiology. 2004, 150, 3463-3472. [CrossRef]

38. Shen, B.; Hutchinson, C.R. Enzymatic synthesis of a bacterial polyketide from acetyl and malonyl coenzyme A. Science 1993, 262, 1535-1540. [CrossRef] [PubMed]

39. Cummings, M.; Breitling, R.; Takano, E. Steps towards the synthetic biology of polyketide biosynthesis. FEMS Microbiol. Lett. 2014, 351, 116-125. [CrossRef] [PubMed]

40. Ryu, Y.-G.; Butler, M.J.; Chater, K.F.; Lee, K.J. Engineering of primary carbohydrate metabolism for increased production of actinorhodin in Streptomyces coelicolor. Appl. Environ. Microbiol. 2006, 72, 7132-7139. [CrossRef] [PubMed]

41. Zabala, D.; Braña, A.F.; Flórez, A.B.; Salas, J.A.; Méndez, C. Engineering precursor metabolite pools for increasing production of antitumor mithramycins in Streptomyces argillaceus. Metab. Eng. 2013, 20, 187-197. [CrossRef]

42. Dayem, L.C.; Carney, J.R.; Santi, D.V.; Pfeifer, B.A.; Khosla, C.; Kealey, J.T. Metabolic engineering of a methylmalonyl-CoA mutase-epimerase pathway for complex polyketide biosynthesis in Escherichia coli. Biochemistry. 2002, 41, 5193-5201. [CrossRef]

43. Chan, Y.A.; Podevels, A.M.; Kevany, B.M.; Thomas, M.G. Biosynthesis of polyketide synthase extender units. Nat. Prod. Rep. 2009, 26, 90-114. [CrossRef] [PubMed]

44. Hertweck, C. The biosynthetic logic of polyketide diversity. Angew. Chem. Int. Ed. Engl. 2009, 48, 4688-4716. [CrossRef] [PubMed]

(C) 2020 by the authors. Licensee MDPI, Basel, Switzerland. This article is an open access article distributed under the terms and conditions of the Creative Commons Attribution (CC BY) license (http://creativecommons.org/licenses/by/4.0/). 